Pacific Journal of Mathematics

THE VOLUME OF A TOTALLY-GEODESIC HYPERSURFACE IN 


\title{
THE VOLUME OF A TOTALLY-GEODESIC HYPERSURFACE IN A PINCHED MANIFOLD
}

\author{
Nathaniel Grossman
}

We will give necessary conditions for a compact hypersurface to be totally-geodesic in a manifold of strictly positive curvatures. These conditions relate the volume of the hypersurface to the pinching of the manifold. The method consists of obtaining estimates from above and below for the volume of the manifold. Comparison of these estimates gives inequalities for the volume of the hypersurface. The method appears on the surface to apply to totally-geodesic submanifolds of arbitrary codimension with but a little modification. We are grateful to $R$. L. Bishop for pointing out that below the surface are snags that we have not yet been able to avoid.

The existence theory for closed geodesics, highly developed yet still incomplete, stands in sharp contrast to the existence theory for higher-dimensional critical submanifolds of Riemannian manifolds. It has long been known that every compact Riemannian manifold carries a closed geodesic. Of course, if the fundamental group of the manifold is not trivial, every nontrivial homotopy class of closed curves contains at least one closed geodesic.

If the $k^{\text {th }}$ homotopy group of a manifold is not trivial, one might hope analogously for a minimal $k$-sphere in each nontrivial homotopy class. Aside from the obvious (and classical) difficulties with the behavior of the higher-dimensional volume functionals, there is an even more fundamental difficulty, pointed out by Kervaire and Milnor: a nontrivial homotopy class on a smooth manifold may fail to have an embedded smooth $\left(C^{1}\right)$ representative! Part of the difficulty may be side-stepped by working in a larger category. Thus, Almgren has obtained strong results using his theory of varifolds. However, the extremal objects may fail to be manifolds on sets which are small in some measure-theoretic sense.

On the other hand, some positive results are known in the category of manifolds. For example, a holomorphic embedding of a Kähler manifold into a complex projective space (with the canonical FubiniStudy metric) is minimal. But the positive results are still meager.

1. Notation and preliminary matters. All manifolds to be considered will be smooth and all Riemannian metrics will be complete. $M$ will be a manifold of dimension $m$, carrying a Riemannian metric $\langle\cdot, \cdot\rangle$ and associated norm $\|\cdot\|$. Let $N$ be a compact smoothly-embedded 
hypersurface in $M$ with normal bundle $N^{\perp}$ carrying induced norm on the fibers again denoted by $\|\cdot\|$. Let exp be the exponential map of the Levi-Civita connection $\nabla$ of $\langle\cdot, \cdot\rangle$ and denote the restriction of $\exp$ to $N^{\perp}$ by $\exp ^{\perp}$.

Let $U(\delta)=\left\{v \in N^{\perp} \mid\|v\|<\delta\right\}$ and define

$$
\tau_{N}=\sup \left\{\delta \mid \exp ^{\perp} \text { is one-to-one on } U(\delta)\right\} .
$$

We have previously considered the problem of estimating $\tau_{N}$ and obtained the following result:

Lemma 1. ([3, Theorem 2]) Let $M$ have positive sectional curvatures $K \leqq 1$ and let $N$ be a compact totally-geodesic hypersurface. Then $\tau_{N} \geqq \pi / 2$.

(Remark: The proof given in [3] for the above result can be generalized in an obvious fashion to show that the inequality $\tau_{N} \geqq \pi / 2$ persists if $N$ is not necessarily a hypersurface but merely has codimension $<m / 2$. Notice that the definitions of $U(\delta)$ and $\tau_{N}$ make sense for submanifolds of arbitrary codimension.)

We will have need for some properties of the index form. Let $N$ be a totally geodesic hypersurface and let $\sigma$ be an $N$-geodesic; that is, $\sigma$ is a geodesic parametrized by arc-length with $\dot{\sigma}(0) \in N^{\perp}$. If $X$ and $Y$ are vector fields along $\sigma$ and orthogonal to it, the index form of the pair $(X, Y)$ on $(0, r]$ is given by

$$
I(X, Y)=\left.\int_{0}^{r}\left\{\left\langle X^{\prime}, Y^{\prime}\right\rangle-\langle R(\dot{\sigma}, X) \dot{\sigma}, Y\rangle\right\}\right|_{t} d t,
$$

where the prime denotes covariant differentiation along $\sigma$ and

$$
R(U, V)=\left[\nabla_{U}, \nabla_{V}\right]-\nabla_{[U, V]}
$$

is the curvature transformation. A Jacobi field is a vector field $Y$ along $\sigma$ and orthogonal to it satisfying $Y^{\prime \prime}+R(\dot{\sigma}, Y) \dot{\sigma}=0$. If $Y$ is a Jacobi field, then

$$
I(X, Y)=\left.\left\langle X, Y^{\prime}\right\rangle\right|_{0} ^{r}
$$

We use the minimizing properties of Jacobi fields in the following form.

Lemma 2. Let $Y$ be a Jacobi field along $\sigma$ on $[0, r]$ with $Y^{\prime}(0)=0$ and let $X$ be any field orthogonal to $\sigma$ with $X(r)=Y(r)$. Then $I(X, X) \geqq I(Y, Y)$ with equality exactly when $X=Y$.

Proof. If $X \neq Y$, then $X-Y \neq 0 . \quad I$ is positive definite, so 


$$
\begin{aligned}
0 & <I(X-Y, X-Y) \\
& =I(X, X)-2 I(X, Y)+I(Y, Y) \\
& =I(X, X)-\left.2\left\langle X, Y^{\prime}\right\rangle\right|_{0} ^{r}+\left.\left\langle Y, Y^{\prime}\right\rangle\right|_{0} ^{r} \\
& =I(X, X)-2\left\langle X(r), Y^{\prime}(r)\right\rangle+\left\langle Y(r), Y^{\prime}(r)\right\rangle \\
& =I(X, X)-\left\langle Y(r), Y^{\prime}(r)\right\rangle \\
& =I(X, X)-I(Y, Y) .
\end{aligned}
$$

The point $\sigma(r)$ (or $r$ itself) is called focal (resp. conjugate) to $\sigma(0)$ along $\sigma$ if there is a Jacobi field $Y$ along $\sigma$ with $Y^{\prime}(0)=0$ (resp. $Y(0)=0)$ and $Y(r)=0$. Well-known theorems of Myers [5] and Berger [1] show that, if the Ricci curvatures of $M$ satisfy $R i \geqq a^{2}>0$, there is a focal (resp. conjugate) point along each $N$-geodesic at distance at most $\pi / 2 a$ (resp. $\pi / a)$ from $N$.

Finally, let $\omega_{m-1}$ denote the volume of the Euclidean unit sphere $S^{m-1}$ and let

$$
\Omega_{m-1}=\int_{0}^{\pi} \sin ^{m-1} r d r .
$$

2. Volume estimates. We will prove the following.

THEOREM. Let $N$ be a totally-geodesic, compact hypersurface of $M$ and suppose $0<a^{2} \leqq R i$ and $0<K \leqq b^{2}$ on $M$. Then

$1^{\circ}$. volume $N \leqq \omega_{m-1} b / a^{m}$;

$2^{\circ}$. if either

(i) $b^{2} / 4<K \leqq b^{2}$ on $M$, or

(ii) $M$ is orientable, even-dimensional, and $0<a^{2} \leqq K$ on $M$, then

$$
\text { volume } N \geqq \omega_{m-1} a / b^{m} \text {. }
$$

Before giving the proof, it is convenient to state a lemma about $\exp ^{\perp}$. We recall that R. L. Bishop [cf. 2] has considered the behavior of the volume element under the exponential map $\exp _{p}$ at a point $p$ in $M$. Let $\sigma$ be a geodesic parametrized by arc-length with $\sigma(0)=p$ and $\dot{\sigma}(0)=v$. Suppose that $R i \geqq a^{2}>0$ and $0<K \leqq b^{2}$ on $M$. Then Bishop proves that the Jacobian determinant $J(t)$ of $\exp _{p}$ at $t v \in M_{p}$ satisfies

$$
\left(\frac{\sin b t}{b t}\right)^{m-1} \leqq J(t) \leqq\left(\frac{\sin a t}{a t}\right)^{m-1},
$$

at least out to the first conjugate point of $p$. Equality holds only in the case of constant curvatures. Dually one can consider an $\mathrm{N}$-geodesic $\sigma$ with $\dot{\sigma}(0)=v$ and the Jacobian determinant $j(t)$ of $\exp ^{\perp}$ at $t v$. Bounds on the growth of $j(t)$ are given by the following. 
Lemma 3. Suppose $0<a^{2} \leqq R i$ and $0<K \leqq b^{2}$ on $M$. Then

$$
\cos ^{m-1} b t \leqq j(t) \leqq \cos ^{m-1} a t,
$$

at least out to the first focal point of $N$ along $\sigma$.

Proof. Pick $p \in N$ and $\left\{y_{1}, \cdots, y_{m-1}\right\}$ be an orthonormal basis of $N_{p}$. Let $\sigma$ be an $N$-geodesic with $\dot{\sigma}(0) \in N^{\perp}$. Let $E_{1}, \cdots, E_{m-1}$ be parallel extensions of $y_{1}, \cdots, y_{m-1}$ along $\sigma$. Suppose $p$ has no focal points along $\sigma$ for $0<t \leqq r$.

Let $Z_{i}$ be the Jacobi field along $\sigma$ with $Z_{i}^{\prime}(0)=0$ and $Z_{i}(r)=E_{i}(r)$. (Existence of $Z_{i}$ is assured since $r$ is nonfocal.) Then

$$
j(t)=\frac{\left\|Z_{1}(t) \wedge \cdots \wedge Z_{m-1}(t)\right\|}{Z},
$$

where $Z=\left\|Z_{1}(0) \wedge \cdots \wedge Z_{m-1}(0)\right\|$. Notice that $j(0)=1$. Moreover, the values $\left\{Z_{i}(r)\right\}$ are orthonormal. It follows by direct calculation that

$$
\frac{j^{\prime}(r)}{j(r)}=\sum_{i=1}^{m-1}\left\langle Z_{i}(r), Z_{i}^{\prime}(r)\right\rangle
$$

Using the assumptions $0<K \leqq b^{2}$ and $Z_{i}^{\prime}(0)=0$, we obtain

$$
\begin{aligned}
\left\langle Z_{i}(r), Z_{i}^{\prime}(r)\right\rangle & =\left.\int_{0}^{r}\left\{\left\|Z_{i}^{\prime}\right\|^{2}-K\left(\dot{\sigma}, Z_{i}\right)\left\|Z_{i}\right\|^{2}\right\}\right|_{t} d t \\
& \geqq\left.\int_{0}^{r}\left\{Z_{i}^{\prime}\left\|^{2}-b^{2}\right\| Z_{i} \|^{2}\right\}\right|_{t} d t \\
& \geqq\left.\left\langle h E_{i}, h^{\prime} E_{i}\right\rangle\right|_{r},
\end{aligned}
$$

using Lemma 2. Here, $h(t) E_{i}(t)$ corresponds to a "Jacobi field" for the sphere of constant curvature $b^{2}$ with $h(r)=1$ and $h^{\prime}(0)=0$. Thus, $h(t)=\cos b t / \cos b r$. It follows that

$$
\left\langle Z_{i}(r), Z_{i}^{\prime}(r)\right\rangle \geqq-b \tan b r .
$$

Summing,

$$
\frac{j^{\prime}(r)}{j(r)} \geqq-(n-1) b \tan b r,
$$

from which (using $j(0)=1$ )

$$
j(r) \geqq \cos ^{n-1} b r .
$$

Next, using Lemma 2 applied to the Jacobi fields $Z_{i}$ and the vector field $h E_{i}$, 


$$
\left\langle Z_{i}(r), Z_{i}^{\prime}(r)\right\rangle \geqq\left.\int_{0}^{r}\left\{\left(h^{\prime}\right)^{2}-K\left(\dot{\sigma}, E_{i}\right) h^{2}\right\}\right|_{t} d t .
$$

Summing and using $R i \geqq a^{2}>0$,

$$
\begin{aligned}
\frac{j^{\prime}(r)}{j(r)} & \leqq\left.(n-1) \int_{0}^{r}\left\{\left(h^{\prime}\right)^{2}-a^{2} h^{2}\right\}\right|_{t} d t \\
& \leqq-(n-1) a \tan a r,
\end{aligned}
$$

if $h(t)=\cos a t / \cos a r$. Thus, as above,

$$
j(r) \leqq \cos ^{n-1} a r .
$$

This completes the proof of the Lemma.

Proof of the Theorem.

$1^{\circ}$. By a well-known theorem of S. B. Myers [5], the assumption $R i \geqq a^{2}>0$ implies that the diameter of $M$ is at most $\pi / a$. Pick $p$ in $M$ and let $B(\pi / a)$ be the $\pi / a$-ball in $M_{p}$. Using Bishop's estimates,

$$
\text { volume } \begin{aligned}
M & \leqq \int_{B(\pi / a)}\left(\text { Jacobian determinant of } \exp _{p}\right) d B \\
& =\int_{S^{m-1}} \int_{0}^{\pi / a} J(r) r^{m-1} d r d \omega_{m-1} \\
& \geqq \int_{S^{m-1}} \int_{0}^{\pi / a} r^{m-1}\left(\frac{\sin a r}{a r}\right)^{m-1} d r d \omega_{m-1} \\
& =\frac{\omega_{m-1} \Omega_{m-1}}{a^{m}} .
\end{aligned}
$$

Next, $\exp ^{\perp}$ is a bijection on $U(\pi / 2 b)$ by Lemma 1 , so that from Lemma 3 we get

$$
\text { volume } \begin{aligned}
M & \geqq \text { volume }\left(\exp ^{\perp} U(\pi / 2 b)\right) \\
& =\int_{U(\pi / 2 b)}\left(\text { Jacobian determinant of } \exp ^{\perp}\right) d U \\
& =\int_{N}^{\pi / 2 b} j(r) d r d N \\
& \geqq \int_{-\pi / 2 b}^{\pi / 2 b} \int_{-\pi / 2 b}^{\pi} \cos ^{m-1} b r d r d N \\
& =(\text { volume } N) \frac{\Omega_{m-1}}{b} .
\end{aligned}
$$

Comparing the two estimates of volume $M$, we get the first inequality of the theorem.

$2^{\circ}$. If either (i) or (ii) holds, the injectivity radius of $M$ is at least $\pi / b[4]$. Therefore, as in $1^{\circ}$, we estimate 


$$
\text { volume } \begin{aligned}
M & \geqq \int_{S^{m-1}} \int_{0}^{\pi / b} r^{m-1}\left(\frac{\sin b r}{b r}\right)^{m-1} d r d \omega_{m-1} \\
& =\frac{\omega_{m-1} \Omega_{m-1}}{b^{m}} .
\end{aligned}
$$

On the other hand, by Berger's extension [1] of the Rauch Comparison Theorem, every $N$-geodesic must carry a focal point of $N$ at distance at most $\pi / 2 \alpha$. Therefore,

$$
\text { volume } \begin{aligned}
M & \leqq \int_{N} \int_{-\pi / 2 a}^{\pi / 2 a} \cos ^{m-1} a r d r d N \\
& =\frac{\omega_{m-1} \Omega_{m-1}}{b} .
\end{aligned}
$$

Comparing these two estimates for volume $N$, we get the second inequality of the Theorem, thereby completing the proof.

It may be instructive to point out the difficulty which arises in codimension greater than one. Let $N$ be a smoothly embedded submanifold of $M$ of dimension $p$. There is again a normal bundle $N^{\perp}$ and a restriction $\exp ^{\perp}$ of the exponential map to $N^{\perp}$. If $p>m / 2$, Lemma 1 above is still valid. What is needed is an estimate of the growth of the Jacobian determinant of $\exp ^{\perp}$. We might try to imitate the above process, choosing an $N$-geodesic $\sigma, p$ Jacobi fields $Z_{1}, Z_{2}, \cdots, Z_{p}$ along $\sigma$ with $Z_{i}(r)=E_{i}(r)$ and $Z_{i}^{\prime}(0)=0$, and $m-p-1$ Jacobi fields $Z_{p+1}, \cdots, Z_{m-1}$ along $\sigma$ with $Z_{j}(0)=0$ and $Z_{j}^{\prime}(r)=E_{j}(r)$. Unfortunately, one has no control over the interrelations between Jacobi fields of the first type and those of the second. For the calculation to proceed as above, one needs that fields of the first type remain orthogonal to those of the second type and this can not be assured.

\section{REFERENCES}

1. M. Berger, An extension of Rauch's metric comparison theorem, Illinois J. Math. 6 (1962), 700-712.

2. R. L. Bishop and R. J. Crittenden, Geometry of Manifolds, Academic Press, New York, 1964.

3. N. Grossman, Two applications of the technique of length-decreasing variations, Proc. Amer. Math. Soc. 18 (1967), 327-333.

4. W. Klingenberg, Riemannsche Geometrie im Grossen (Lecture notes prepared by

D. Gromoll and W. Meyer), Bonn, 1962.

5. S. B. Myers, Riemannian manifolds with positive mean curvature, Duke Math. J. 8 (1941), 401-404.

Received November 16, 1966. Supported in part by National Science Foundation grant GP-7036. 


\section{PACIFIC JOURNAL OF MATHEMATICS}

\section{EDITORS}

\section{H. ROYDEN \\ Stanford University \\ Stanford, California}

\author{
J. P. JANS \\ University of Washington \\ Seattle, Washington 98105
}

J. DugundJI

Department of Mathematics

Rice University

Houston, Texas 77001

RichaRd ARENS

University of California

Los Angeles, California 90024

\section{ASSOCIATE EDITORS}
E. F. BECKENBACH
B. H. NeumanN
F. WOLF
K. YOSIDA

\section{SUPPORTING INSTITUTIONS}

\author{
UNIVERSITY OF BRITISH COLUMBIA \\ CALIFORNIA INSTITUTE OF TECHNOLOGY \\ UNIVERSITY OF CALIFORNIA \\ MONTANA STATE UNIVERSITY \\ UNIVERSITY OF NEVADA \\ NEW MEXICO STATE UNIVERSITY \\ OREGON STATE UNIVERSITY \\ UNIVERSITY OF OREGON \\ OSAKA UNIVERSITY \\ UNIVERSITY OF SOUTHERN CALIFORNIA
}

\author{
STANFORD UNIVERSITY \\ UNIVERSITY OF TOKYO \\ UNIVERSITY OF UTAH \\ WASHINGTON STATE UNIVERSITY \\ UNIVERSITY OF WASHINGTON

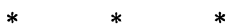 \\ AMERICAN MATHEMATICAL SOCIETY \\ CHEVRON RESEARCH CORPORATION \\ TRW SYSTEMS \\ NAVAL ORDNANCE TEST STATION
}

Mathematical papers intended for publication in the Pacific Journal of Mathematics should be typewritten (double spaced). The first paragraph or two must be capable of being used separately as a synopsis of the entire paper. It should not contain references to the bibliography. Manuscripts may be sent to any one of the four editors. All other communications to the editors should be addressed to the managing editor, Richard Arens at the University of California, Los Angeles, California 90024.

50 reprints per author of each article are furnished free of charge; additional copies may be obtained at cost in multiples of 50 .

The Pacific Journal of Mathematics is published monthly. Effective with Volume 16 the price per volume (3 numbers) is $\$ 8.00$; single issues, $\$ 3.00$. Special price for current issues to individual faculty members of supporting institutions and to individual members of the American Mathematical Society: $\$ 4.00$ per volume; single issues $\$ 1.50$. Back numbers are available.

Subscriptions, orders for back numbers, and changes of address should be sent to Pacific Journal of Mathematics, 103 Highland Boulevard, Berkeley 8, California.

Printed at Kokusai Bunken Insatsusha (International Academic Printing Co., Ltd.), 7-17, Fujimi 2-chome, Chiyoda-ku, Tokyo, Japan.

PUBLISHED BY PACIFIC JOURNAL OF MATHEMATICS, A NON-PROFIT CORPORATION

The Supporting Institutions listed above contribute to the cost of publication of this Journal, but they are not owners or publishers and have no responsibility for its content or policies. 


\section{Pacific Journal of Mathematics}

\section{Vol. 23, No. 2 \\ April, 1967}

Herbert Stanley Bear, Jr. and Bertram John Walsh, Integral kernel for

one-part function spaces .......................... 209

Mario Borelli, Some results on ampleness and divisorial schemes ....... 217

John A. Erdos, Unitary invariants for nests . ................... 229

Nathaniel Grossman, The volume of a totally-geodesic hypersurface in a pinched manifold.................................. 257

D. M. Hyman, A generalization of the Borsuk-Whitehead-Hanner theorem ............................................. 263

I. Martin (Irving) Isaacs, Finite groups with small character degrees and large prime divisors ............................. 273

I. Martin (Irving) Isaacs, Two solvability theorems ................ 281

William Lee Johnson, The characteristic function of a harmonic function in a locally Euclidean space ............................... 291

Ralph David Kopperman, Application of infinitary languages to metric spaces ............................................. 299

John Lauchlin MacDonald, Relative functor representability ............ 311

Mahendra Ganpatrao Nadkarni, A class of measures on the Bohr group.... 321

Keith Lowell Phillips, Hilbert transforms for the p-adic and p-series fields....................................... 329

Norman R. Reilly and Herman Edward Scheiblich, Congruences on regular semigroups ...

Neil William Rickert, Measures whose range is a ball ... . .

Gideon Schwarz, Variations on vector measures

Ronald Cameron Riddell, Spectral concentration for self-adjoint operators. .

Haskell Paul Rosenthal, A characterization of restrictions of

Fourier-Stieltjes transforms ................... 